\title{
Promoting Shared Decision-Making in Rehabilitation: Development of a Framework for Situations When Patients with Dysphagia Refuse Diet Modification Recommended by the Treating Team
}

\author{
Franceen Kaizer • Anna-Maria Spiridigliozzi • \\ Matthew R. Hunt
}

Received: 5 May 2010/Accepted: 26 March 2011/Published online: 1 May 2011

(C) Springer Science+Business Media, LLC 2011

\begin{abstract}
To address the risks of aspiration pneumonia, patients with dysphagia may be prescribed a modified diet. The goal of diet modification is to decrease the risk of patients aspirating food due to their diminished swallowing reflex. Some patients may not accept diet modification or may not adhere to the treatments identified by the interdisciplinary team. Such scenarios may result in important moral uncertainty and concern for clinicians. As a result of several ethics consultations related to this issue, a working group of the Clinical Ethics Committee at the Jewish Rehabilitation Hospital in Laval, Quebec, Canada, developed a framework for responding to situations when patients do not adhere to recommended diet modification. The goal of this tool is to facilitate discussion and collaboration between clinicians and patients, to clarify assumed versus real risk, and to promote shared decision-making in dysphagia care. In this article we examine the clinical context of diet modification for patients with dysphagia in rehabilitation hospitals, explore ethical aspects of this topic, present the clinical algorithm, and discuss our experience with developing and piloting this tool.
\end{abstract}

Keywords Dysphagia - Shared decision-making · Diet modification - Ethics - Rehabilitation - Adherence · Deglutition · Deglutition disorders

F. Kaizer $(\bowtie)$ - A.-M. Spiridigliozzi

Jewish Rehabilitation Hospital, 3205, Place Alton-Goldbloom, Laval, QC H7V 1R2, Canada

e-mail: franceen_kaizer@ssss.gouv.qc.ca

M. R. Hunt

Center for Research on Ethics, University of Montreal

(CREUM), C.P. 6128, succ. Centre-ville, Montreal,

QC H3C 3J7, Canada
Dysphagia, described as a combination of symptoms affecting a person's ability to swallow, is a frequent finding in patients admitted for intensive rehabilitation, particularly among those admitted following acute stroke. In a review of the literature, Martino et al. [1], reported frequencies of dysphagia following stroke ranging from 19 to $81 \%$, depending on study design, patient characteristics, and stroke type. Many patients regain their swallowing abilities shortly after the onset of the stroke [2], but some have persistent difficulties upon being transferred to rehabilitation. Other conditions with associated dysphagia include multiple sclerosis, Guillain-Barré, head and neck cancer, and Alzheimer's disease. A range of potential complications of dysphagia has been documented and includes chest infections, malnutrition, and increased mortality [3]. Patients with dysphagia tend to have decreased functional status, slower recovery [3], and, subsequently, longer lengths of stay in hospital. These findings have prompted health-care professionals to develop dysphagia management strategies to minimize these adverse outcomes.

The dysphagia management process begins with an interdisciplinary assessment from which a treatment plan is developed with the goal of minimizing the risk of aspiration pneumonia, malnutrition, and dehydration. Treatment recommendations may include postural adjustment, swallowing manoeuvres, and modifications in food consistency. Diet modification of liquids, solids, or both is a common strategy used in the management of dysphagia. The success of these recommendations requires considerable patient participation and compliance.

Many patients capable of self-feeding follow the recommendations developed by the treatment team. However, the results of one study [4] suggest that as many as $40 \%$ do not. Low et al. [5] note that there is a correlation between 
the level of compliance and the type of advice given. In their study, patients were more likely to adhere to recommendations regarding modification of solids than with recommendations of liquid modification. Other research has demonstrated that stroke patients with dysphagia rarely perceive that they have a swallowing problem [6], a situation that adds to the challenge of patient education and the promotion of adherence with recommended diet modifications.

Noncompliance with recommendations about dysphagia management is associated with adverse outcomes [5]. Numerous studies have examined reasons for noncompliance; however, most of these studies, conducted in acute care and nursing home settings, considered that the sources of noncompliance with the diet modifications prescribed for hospitalized patients were hospital staff [7-9]. These findings showed that the reasons for failure by hospital nursing staff to adhere to diet modifications included lack of knowledge regarding dysphagia, lack of time, and disagreement with the recommendations.

Patients in an active rehabilitation setting are often faced with numerous challenges and experience many significant changes and losses. Some patients struggle to understand the nature and implications of changes that may include increased dependence, uncertainty regarding return home or to work, changing social and family roles, and reassessment of life plans. Patient participation and engagement in the decision-making process are key to ensuring the most successful outcome of rehabilitation care possible. Clinicians aim to support and promote the decision-making of patients. However, challenging situations arise when patients, for a variety of reasons, do not follow the recommendations made by the treatment team. In such situations, institutional and policy supports can assist clinicians in their work with patients. To date, however, there has been limited discussion of practical approaches in the literature when hospitalized patients (and their families) refuse or do not adhere to diet texture modifications.

\section{Ethical Considerations}

An important source of ethical concern for health-care professionals in rehabilitation institutions is when patients wish to make choices that clinicians assess as unduly risky [10]. The refusal of treatment recommendations by patients with dysphagia admitted to a rehabilitation hospital is a prominent example of such issues. Such situations may be sources of moral unease or distress for health-care professionals [11]. These concerns may be elevated if healthcare professionals feel that they are active participants in an activity that places the patient at risk of serious harm. For example, hospital staff may need to facilitate feeding that they feel is unsafe. Some health providers may feel responsible if negative consequences result for the patient. On the other hand, patients and families may feel that they are being forced to follow a dysphagia management plan that they do not understand or agree with. This issue can also create divisions among family members who hold different views on what the best course of action is for their loved one. Divergent views regarding diet modification can strain the therapeutic relationship between patients/families and the treating team, and hamper efforts toward shared decision-making. Sharp notes that the "clear link between dysphagia and the potential for serious complications or death can produce intensely emotional responses among team members and the patient's family" [12]. This situation is compounded due to the challenges associated with risk assessment and communication of risk in health-care settings $[13,14]$.

A primary ethical consideration that is relevant to the refusal of treatment recommendations by patients with dysphagia is the principle of respect for autonomy. Autonomy is typically understood as an individual's right and ability to make choices and practice self-determination [15]. Respect for autonomy also places a negative duty on others not to interfere in the exercise of an individual's autonomy without compelling reasons for such interference. In health care, autonomy is closely linked to the doctrine and practice of informed consent. Informed consent is a process by which a competent patient, or the duly identified surrogate decision-maker for an incompetent patient, is provided with the opportunity to receive relevant information regarding a medical decision, ask questions, weigh options, and enact decisions that are consistent with their goals and values, and free from coercion. In a range of circumstances, respecting a competent patient's informed refusal of an offered treatment is consistent with the principle of autonomy.

Commentators on autonomy have also focused on the important roles that health-care providers play in seeking to support and promote the autonomy of patients [16]. Feminist bioethicists encourage a broader conception of autonomy that acknowledges and accounts for interdependencies and relationships [17, 18]: this approach emphasizes that individuals are embedded in social and relational webs of significance and support. A relational conception of autonomy suggests that efforts be made so that treatment decision-making does not result in further isolating the patient but rather, to the extent possible, health-care professionals, family members, and friends should support patients to make choices that are consistent with the patient's sense of what is important to her or him and what makes sense for her or his life.

Autonomy does not capture the entirety of what is ethically important in such situations. Health-care professionals 
also seek to promote the well-being of the patient and are guided by the principle of beneficence - to act for the benefit of others, specifically to do good for patients in their care. Furthermore, health-care professionals endeavour to minimize the likelihood of harm for patients. In regard to treatment decisions, health-care professionals provide education to patients regarding the implications of different options and seek to guide patients toward decisions that are most likely to result in benefit and minimize the risk of harm. Shared decision-making involves active participation of the treatment team in working with patients (and sometimes family members or friends) to make decisions. In situations where a patient wishes to make a choice that is understood by the clinician to be unduly risky (such as to forgo treatment that has a high likelihood of benefit and without which harm is likely to result), it is consistent with this approach for healthcare professionals to begin to clarify the implications of a decision for the patient and attempt to understand the issue from the patient's perspective. In addition, taking into consideration the patient's rationale for a decision and the meaning it holds for him or her, health-care professionals are justified in seeking to actively dissuade patients from making choices or performing actions that expose the patient (or others) to harms which are not offset by potential benefits and are inconsistent with the goals of care. Health-care professionals should be careful to avoid using coercion or imposing their own values and assumptions on the evaluation of risk and potential benefit.

This brief sketch is intended to highlight some key ethical considerations related to decision-making regarding diet modification for patients with dysphagia. In the following section we describe our experiences at a rehabilitation hospital in creating supports for shared decisionmaking in dysphagia management.

\section{Dysphagia Management at the Jewish Rehabilitation Hospital}

The Jewish Rehabilitation Hospital (JRH) is a 126-bed rehabilitation hospital in Laval, Quebec, affiliated with McGill University. Rehabilitation programs include Neurology, Traumatic Brain Injury, Geriatrics, Pulmonary, Oncology, and Orthopaedics. There are approximately 1,100 patients admitted each year for inpatient rehabilitation. As many as $41 \%$ of patients admitted to the Neurology Program are identified as having a swallowing disorder. Other programs admit patients with dysphagia, with rates from 5 to $17 \%$.

In 2002 the hospital created an interdisciplinary dysphagia management committee to develop practice guidelines and support clinicians in the management of patients with dysphagia. The goals of dysphagia management at the JRH are (1) to identify clients with swallowing and chewing disorders; (2) to prevent complications such as aspiration pneumonia, malnutrition, and dehydration; (3) to help the patient achieve safety, adequacy, and independence in swallowing, chewing, and overall oral feeding; and (4) to educate staff, family, and patient about the nature and management of swallowing disorders. The core JRH dysphagia team consists of a dietician, nurse, occupational therapist, physician, and speech language pathologist. Other team members (e.g., a pharmacist, physiotherapist, psychologist, respiratory therapist, and social worker) are involved in dysphagia management as needed.

Dysphagia management at the JRH has evolved over the past two decades. This has occurred in response to a significant increase in the number of patients with dysphagia treated at the hospital. Alongside clinical developments, the team has also addressed questions related to confidentiality, autonomy, patient/family wishes, quality of life, and adherence. In our experience, questions related to nonadherence have been an important source of discussion and sometimes discomfort for the team. Concerns have been related to the patient's well-being, feelings of professional ineffectiveness or inadequacy because recommendations were not followed, and concern about their own professional and legal obligations in the event that the patient has important medical complications because of their nonadherence. There have also been situations when individual team members experienced an enormous sense of guilt and responsibility when they participated in treatment activities that challenged their own personal and professional values. For example, a nurse fed a non-recommended texture to a patient (following refusal of the modified diet by the patient) despite her strong conviction that this action was wrong because it might have negative consequences on his health. Unfortunately, in many situations clinicians felt that they were unable to share their feelings and experienced isolation and anxiety.

Initially, the team looked for quick fixes to these issues, such as requesting that the patient/family sign an informed refusal form or a waiver of responsibility. However, it became clear that the treatment team needed a systematic and structured approach to assist them in dealing with issues of patient/family non-adherence in an efficient manner. This is consistent with Sharp's assertion that institutional policies for the refusal of diet modification will assist patients, families, and clinicians to respond to such situations [12].

Following discussion with the Clinical Ethics Committee, a working group was formed to create a clinical guideline entitled "Clinical Decision Making Process for Dysphagia Diet Modifications" (Fig. 1). The objective of 
Fig. 1 Clinical decision making process for Dysphagia diet modifications

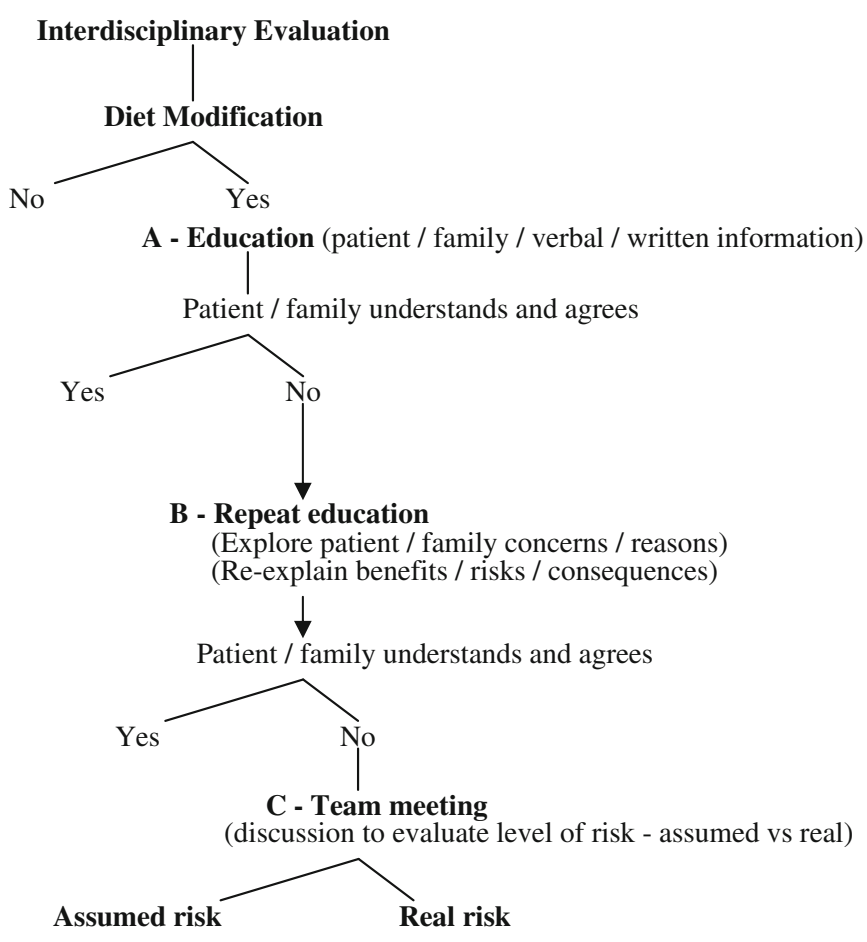

Team plan which may include:

1. Altering diet modification

2. Maintain diet modification knowing patient / family are not respecting it

3. Teaching \& reinforcing safety precautions

4. Shared responsibility of team and family

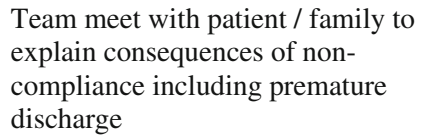

Team meet with patient / family to explain consequences of noncompliance including premature discharge

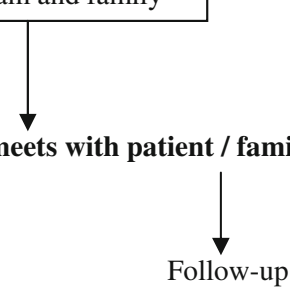

this tool was to provide structured guidelines for the clinical teams to follow when patients and families refused to follow recommendations. This tool aimed to support team members in developing, modifying as necessary, and following through on treatment plans.

\section{Development of Dysphagia Algorithm to Promote Shared Decision-making}

At an early stage of developing this tool, agreement was sought on underlying principles and approaches. A range of principles and key considerations was identified: The tool needed to reflect the collective and shared values of the team and the hospital and also be consistent with patient rights and responsibilities, a patient-centered care approach, and the rehabilitation goal of maximizing the patient's functional autonomy. The tool needed to be clear, concise, readily accessible, and relevant for the clinical teams. Finally, it was noted that the guidelines should facilitate team discussions in a structured and guided manner, promote improved dysphagia management, enhance support amongst the team, and promote effective communication.

An important consideration addressed in creating the tool was the concept of what quality of life meant to different people involved in the process of managing dysphagia, including the patient and his or her family and individual team members. The significance of eating in relation to quality of life varies from individual to individual. Cultural and social values and expectations may be important factors in how individuals view decisions related to diet modification. Such considerations are critical components of discussions leading to the development of a treatment plan. Upon completion, the algorithm was reviewed by the Clinical Ethics Committee and several 
clinical teams. Feedback and comments were integrated into the final version of the document.

The tool that was developed provides a framework for considering the needs, interests, and perspectives of individual patients in their particularity, and avoids viewing cases in an abstract or inconsistent manner. A key element of the tool is a focus on eliciting and considering the reasons why a patient refuses or does not adhere to the treatment recommendations.

In addition, the tool was designed to promote reflection around evaluations of risk. A distinction is drawn between real and assumed risks. Real risk is defined as situations in which there are clinical, measurable signs that non-adherence to the dysphagia recommendations are negatively affecting the patient's health. These signs include history or presence of aspiration pneumonia, weight loss, chronic obstructive pulmonary disease (COPD), reduced oxygen saturation, signs of dehydration, or fever. Assumed risk is defined as the potential for a negative outcome that may seriously impact on the person's health status. Distinguishing real and assumed risk has proven to be very beneficial. This aspect of the tool assists clinicians in objectively basing their decision about risk for aspiration on clinical signs.

\section{The Dysphagia Algorithm}

The dysphagia algorithm is reproduced in Fig. 1. Once the interdisciplinary dysphagia evaluation is complete and a recommendation for a modified diet texture is made, a designated team member meets with the patient and family to explain the evaluation results and recommendations. Patient and family education has always been an element of clinical practice; however, the guidelines provide a structured approach to teaching and education (A). Providing simple and clear written educational material in addition to verbal communication regarding recommendations is encouraged. Whenever possible, the team must seek to ensure that the information is not only heard but understood. Discussions with the patient and family address issues such as benefits, risks, and consequences of dysphagia diet modification as well as alternatives. This practice permits the team and the patient/family to discuss issues, answer questions, share concerns, and negotiate an acceptable common ground that promotes adherence to the treatment plan.

With this approach, it is insufficient to inform the patient of the recommended diet texture and document that the patient is noncompliant should they refuse. Rather, the guidelines call for repeated and ongoing exchanges between the team members and the patient and family. During this process, it is critical that the team examine why the patient is refusing or not adhering to the treatment recommendations made by the team (B).

If after these initial steps the patient and/or family continue to refuse the recommended diet modifications, additional efforts are to be made to explore patient/family concerns and understand the situation and the rationale for their decision. The importance of involving the family cannot be underestimated. Many patients with dysphagia do not understand and have difficulty following recommendations due to cognitive impairments, and stroke patients with dysphagia are often not aware that they have a swallowing problem [6]. If the patient continues to refuse the diet modification, a team meeting is organized (C), with the objective of deciding whether to maintain the recommendation knowing that the patient is not complying, or consider modifying the recommendations in view of the level of risk. Distinguishing assumed versus real risk plays an important part in this discussion. In situations when the patient's health and rehabilitation potential are being seriously compromised due to real risk of harm, elevated in both likelihood and magnitude, the guidelines allow for premature discharge. Following the team meeting, the team meets with the patient/family to share the proposed treatment plan and outline any changes, again emphasizing safety precautions. The team reinforces the notion of shared responsibility in the development of the final treatment plan.

Documentation is an integral component of the tool at each stage in the process. In addition to helping assure that all the steps of the algorithm are followed, ongoing documentation provides a valuable mechanism for communication required amongst all team members.

\section{Implementation}

The tool was presented to the health-care professionals involved in the treatment of dysphagia. There was phased implementation over the summer of 2007, starting with the Neurology unit, where most of the patients with dysphagia are admitted.

During the period 2008-2010, 258 patients were treated for dysphagia and subsequently discharged from the hospital. Of those, 16 were identified as having some issues related to compliance during their hospitalization. We conducted a review of these 16 charts. What was evident from the review was the extended negotiation and discussion that took place in each of these cases. There were three types of outcomes following education, discussion, and negotiation as outlined in the algorithm: reluctant acceptance of the team's recommendation by the patient and family ( 9 cases), minor modification of the treatment plan (compromise) with close supervision and frequent bedside 
re-evaluation (4 cases), and continued refusal to adhere to recommendations (3 cases). One patient, though deemed competent for decision-making, presented with cognitive problems and categorically refused the team's recommendations to not drink thin liquids. This patient was ambulatory and frequented the cafeteria on his own. The team monitored him closely with frequent bedside re-evaluations until he progressed to being able to safely drink thin liquids. The second patient presented with a severe dysphagia and a feeding tube had been inserted prior to his admission to the rehabilitation hospital. Upon evaluation, the team recommended no oral feeding. His wife insisted on feeding him orally despite repeated education regarding the elevated risks. Trials of food were done and unfortunately the patient developed aspiration pneumonia and was transferred to acute care. Upon the patient's readmission to rehabilitation, a meeting was held with the patient's wife. Following detailed explanations and discussions (as outlined in level B of the algorithm), she was more accepting of the team's recommendations. The third patient insisted on drinking water despite repeated education and categorically refused the team's request to include his wife in discussions about his swallowing difficulties. The patient insisted that he was willing to accept the risks associated with not following the recommendations. Following a new bedside swallowing evaluation and team discussion, the team accepted the patient's decision regarding his diet, monitoring closely with frequent re-evaluations. He eventually progressed and was considered able to drink regular liquids safely upon discharge. In these three cases issues of compliance were not resolved; however, following the algorithm ensured that the team's approach was thorough, substantial teaching and discussion were conducted, the process was well-documented, and the clinician's felt supported despite the challenging nature of these cases. Although premature discharge is a possibility allowed for in the guidelines, to date it has not been implemented at the JRH. Alternatives and compromise have always led to other ways to resolve concerns.

The introduction of the dysphagia algorithm has brought about a change in clinical practice. With an increased emphasis on patient education throughout the process of evaluation and treatment and the concept of shared responsibility, clinicians report that they are more comfortable in providing their recommendations and that they receive less resistance from patients and families.

As seen in 13 of the 16 charts reviewed, issues of compliance were resolved using the principles and steps outlined in the algorithm. At this time it is not possible to determine how many patients would have presented with issues of compliance or adherence had the algorithm not been in place. Since implementing the algorithm, greater detail in documentation of discussions and decision-making processes has now become standard practice for dysphagia management. Comparison with how decisions were made in cases prior to the implementation was not feasible from chart review because documentation of these cases was less detailed. In this article we have presented our experiences using the algorithm with 16 patients with dysphagia admitted to a rehabilitation hospital. Systematic evaluation of the use of the algorithm with a larger number of cases would support assessment of the tool's effectiveness.

\section{Conclusion}

Shared decision-making is an important principle of rehabilitation practice. This approach is consistent with patientcentered care. The management of dysphagia, and in particular the refusal of diet modification by patients with dysphagia, can create challenges for implementing shared decision-making. To support clinicians and promote shared decision-making, an algorithm was created to address situations of refusal or non-adherence to diet modification recommended for patients with dysphagia admitted to an inpatient rehabilitation unit.

We note that the guidelines have become integrated into standard practice as the team refers less to the tool but incorporates the principles and steps when elaborating a dysphagia treatment plan involving diet modifications. In our experience, the utilization of the algorithm has improved team communication with the patient and family and provided a means for team members to feel supported and guided when dealing with non-adherence issues in dysphagia.

Acknowledgments Members of the dysphagia working group that developed the algorithm include Franceen Kaizer, Sandra Prizio, and Anna-Maria Spiridigliozzi. We thank Jane Chambers Evans and the members of the Jewish Rehabilitation Hospital Clinical Ethics Committee for their valuable input during the development of the algorithm. We also thank Annie Dicaire for her editorial assistance and Irene Shanefield for her contribution to the literature review. Matthew Hunt's research is supported by a postdoctoral fellowship from the Canadian Institutes of Health Research.

\section{References}

1. Martino R, Foley N, Bhogal S, Diamant N, Speechley M, Teasell R. Dysphagia after stroke: incidence, diagnosis, and pulmonary complications. Stroke. 2005;36:2756-63.

2. Smithard DG, O'Neill PA, England RE, Park CL, Wyatt R, Martin DF, Morris J. The natural history of dysphagia following a stroke. Dysphagia. 1997;12:188-93.

3. Smithard DG, O'Neill PA, Parks C, Morris J. Complications and outcome after acute stroke. Does dysphagia matter? Stroke. 1996;27:1200-4. 
4. Sharp HM, Bryant KN. Ethical issues in dysphagia: when patients refuse assessment or treatment. Semin Speech Lang. 2003;24(4): 285-99.

5. Low J, Wyles C, Wilkinson T, Sainsbury R. The effect of compliance on clinical outcomes for patients with dysphagia on videofluoroscopy. Dysphagia. 2001;16:123-7.

6. Parker C, Power M, Hamdy S, Bowen A, Tyrrell P, Thompson DG. Awareness of dysphagia by patients following stroke predicts swallowing performance. Dysphagia. 2004;19:28-35.

7. Rosenvinge SK, Starke ID. Improving care for patients with dysphagia. Age Ageing. 2005;34:587-93.

8. Colodny ED. Construction and validation of the mealtime and dysphagia questionnaire: An instrument designed to assess nursing staff reasons for non-compliance with SLP dysphagia and feeding recommendations. Dysphagia. 2001;16:263-71.

9. McCullough KC, Estes JL, McCullough GH, Rainey J. RN compliance with SLP dysphagia recommendations in acute care. Top Geriatr Rehabil. 2007;23(4):330-4.

10. Hunt MR, Ells C. Partners towards autonomy: relational autonomy and risky choices in rehabilitation care. Disabil Rehabil. 2011. http://informahealthcare.com/doi/abs/10.3109/09638288. 2010.515703.

11. Browne A. Helping residents live at risk. Camb Q Healthc Ethics. 2003;12:83-90.

12. Sharp HM. Ethical issues in the management of dysphagia after stroke. Top Stroke Rehabil. 2006;13(4):18-25.
13. Banja JD. Risk assessment and patient autonomy. J Head Trauma Rehabil. 1994;9(4):70-2.

14. Macciocchi SN, Stringer AY. Assessing risk and harm: the convergence of ethical and empirical considerations. Arch Phys Med Rehabil. 2001;82(Suppl 2):S15-9.

15. Beauchamp TL, Childress JF. Principles of biomedical ethics, 6th edn. New York: Oxford University Press; 2009.

16. Bergsma J, Thomasma DC. Autonomy and clinical medicine: renewing the health professional relation with the patient. Dordrecht: Kluwer Academic Publishers; 2000.

17. Sherwin S. A relational approach to autonomy in health care. In: Sherwin S, editor. The politics of women's health: exploring agency and autonomy. Philadelphia: Temple University Press; 1998. p. 19-47.

18. Donchin A. Understanding autonomy relationally: toward a reconfiguration of bioethical principles. J Med Philos. 2001; 26(4):365-86.

Franceen Kaizer BSc

Anna-Maria Spiridigliozzi MSc

Matthew R. Hunt $\mathrm{PhD}$ 